Special issue of the 2nd International Conference on Computational and Experimental Science and Engineering (ICCESEN 2015)

\title{
Theoretical Production of Radiopharmaceuticals Used in Mycardial Perfusion Scintigraphy
}

\author{
N. KARPUZ ${ }^{a, *}$ AND B. MAVI ${ }^{b}$ \\ ${ }^{a}$ Amasya University, Sabuncuoğlu Şerefeddin Health Services Vocational School, Amasya, Turkey \\ ${ }^{b}$ Amasya University, Sciences \& Arts Faculty, Amasya, Turkey
}

\begin{abstract}
Myocardial perfusion scintigraphy, is an imaging technique used for diagnostics of coronary artery disease in people by evaluating the amount of blood reaching the heart muscle. During myocardial perfusion scintigraphy a radiopharmaceutical is injected into the patient and after a period of time a series of images are acquired using gamma camera rotated by $180^{\circ}$ around the patient. The distribution of the radiopharmaceutical is then determined using the technology of single photon emission computed tomography. The most commonly used agents in myocardial perfusion scintigraphy radiopharmaceuticals are Tl-201 and Tc-99m. The production of such radiopharmaceuticals containing Tl-201 and Tc-99m has been examined by this study. This study was performed using Monte Carlo nuclear reaction simulation code TALYS 1.6.
\end{abstract}

DOI: 10.12693/APhysPolA.130.304

PACS/topics: 13.85.Lg, 25.60.Dz

\section{Introduction}

Nuclear reactions induced by incident intermediate and high energy protons are very important because of wide range of technical applications [1]. Nowadays, lots of radioisotopes are used in medical applications. The radioisotope production can be performed using $(\mathrm{p}, \mathrm{x}),(\mathrm{n}, \mathrm{x})$ reactions.

Radioisotopes used in medicine are produced using both nuclear reactors and cyclotrons [2]. In the radioisotope production programs, nuclear reaction data are mainly needed for optimization of production routes. This process involves a selection of the projectile energy range that will maximize the yield of the product and minimize that of the radioactive impurities [3]. Nuclear reactions calculations are based on standard nuclear reaction models and they can be helpful for determining the accuracy of various parameters of nuclear models and experimental measurements.

Calculations which are based on nuclear reaction models play an important role in the investigation of reaction cross sections [4]. Therefore, data on the cross section of reactions, induced by both protons and neutrons, are essential for radioisotope production.

Cardiac nuclear medicine studies play important roles in the diagnosis, clinical making and follow-up of various cardiac diseases. Myocardial perfusion scintigraphy provides significant knowledge to determine myocardial ischemia and viability in patients with both known and suspected coronary artery disease, and to assess risk stratification and prognostic evaluation as well. Myocardial perfusion (blood supply) scintigraphy principle is based on the principle of radiopharmaceuticals keeping

\footnotetext{
*corresponding author; e-mail: nurdankarpuz@gmail.com
}

hold of the heart muscle. Thallium-201 (Tl-201, $t_{1 / 2}=$ $72.91 \mathrm{~h})$ and technetium- $99 \mathrm{~m}\left(\mathrm{Tc}-99 \mathrm{~m}, t_{1 / 2}=6.02 \mathrm{~h}\right)$ are the most commonly used radioactive substances for this purpose.

\section{Method and figures}

Nowadays, myocardial perfusion imaging using single photon emission computed tomography, is probably the most common practice in nuclear cardiology. It is based on evaluation of the myocardial blood flow for the diagnosis and prognosis of ischaemic heart disease. Myocardial perfusion can be performed with many radioactive perfusion tracers and tomographic imaging techniques. Tl-201 and Tc-99 are commonly used radioactive tracers for myocardial perfusion.

Therefore in this study, production cross section for Tl201 and Tc-99m were calculated using nuclear reaction simulation code TALYS $1.6[5]$ and compared to existing experimental data in EXFOR [6] data file.

TALYS is a nuclear reaction simulation computer code system for the analysis and prediction of nuclear reactions. The TALYS is a Fortran-based program and it can be run under Linux system. It can analyse many different kinds of nuclear reaction types for target nucleus with the mass number of five or heavier. The energy range for incoming particle is in the range of $1 \mathrm{keV}-$ $250 \mathrm{MeV}$ for neutron, proton, deuteron, triton, $3 \mathrm{He}$, alpha particle and photon. The basic objective behind its construction is the simulation of nuclear reactions that involve neutrons, photons, protons, deuterons, tritons, $3 \mathrm{He}$-and alpha particles. TALYS integrates the optical model, direct, preequilibrium fission and statistical nuclear reaction models in one calculation scheme and gives a prediction for all open reaction channels.

${ }^{201} \mathrm{Tl}$ and ${ }^{99 m} \mathrm{Tc}$ production cross section values for the reaction were calculated in the energy range between 5 and $130 \mathrm{MeV}$. 


\subsection{Figures}

The calculated ${ }^{203} \mathrm{Tl}(\mathrm{p}, \mathrm{n}){ }^{201} \mathrm{~Pb}, \quad{ }^{201} \mathrm{~Pb}-{ }^{201} \mathrm{Tl}$ and ${ }^{100} \mathrm{Mo}(\mathrm{p}, 2 \mathrm{n}){ }^{99 m} \mathrm{Tc}$ production reaction cross sections are shown in Figs. 1 and 2. The obtained results have been compared with the experimental data existing in the EXFOR databases.

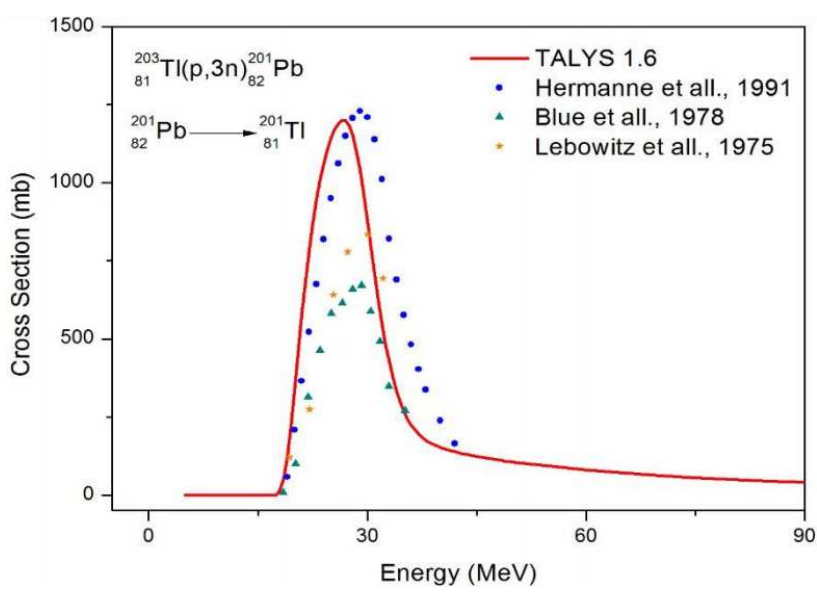

Fig. 1. ${ }^{203} \mathrm{Tl}(\mathrm{p}, 3 \mathrm{n}){ }^{201} \mathrm{~Pb},{ }^{201} \mathrm{~Pb}-{ }^{201} \mathrm{Tl}$ production reaction cross section.

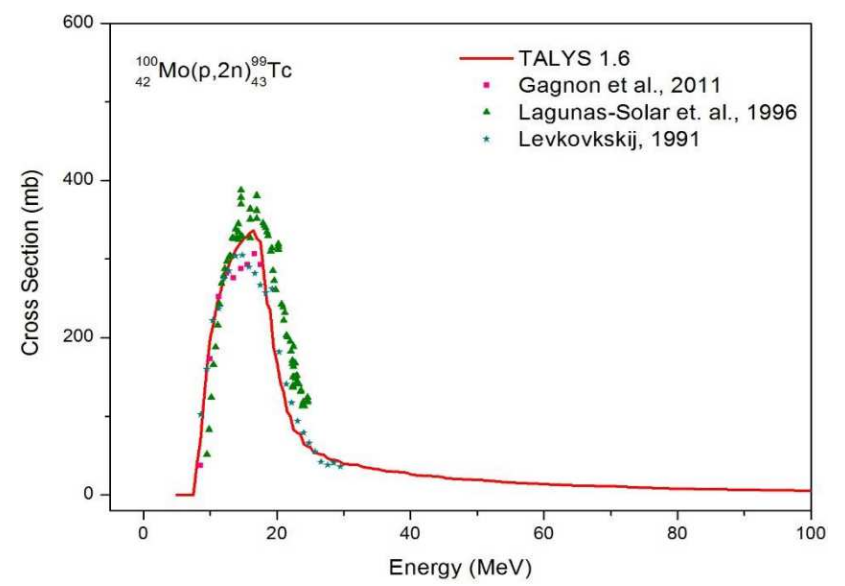

Fig. 2. ${ }^{100} \mathrm{Mo}(\mathrm{p}, 2 \mathrm{n})^{99} \mathrm{Tc}$ production reaction cross section.

\section{Conclusions}

In this work, ${ }^{203} \mathrm{Tl}(\mathrm{p}, 3 \mathrm{n}){ }^{201} \mathrm{~Pb} ; \quad{ }^{201} \mathrm{~Pb}-{ }^{201} \mathrm{Tl}$ and ${ }^{100} \mathrm{Mo}(\mathrm{p}, 2 \mathrm{n})^{99 m} \mathrm{Tc}$ production cross section values for the reactions were calculated in the energy range between 5 and $130 \mathrm{MeV}$. Our results are in fair agreement with available experimental data in literature. The maximum cross section values are $336.15 \mathrm{mb}$ at $16.5 \mathrm{MeV}$ for ${ }^{99} \mathrm{Tc}$ production and $1200.36 \mathrm{mb}$ at $27 \mathrm{MeV}$ for ${ }^{201} \mathrm{Tl}$ production. Values predicted by TALYS 1.6 are quite lower when compared to our data, in the energy range covered in this work. It can be seen that optimum energy range for production of ${ }^{201} \mathrm{Tl}$ with accelerated proton particles is $16-42 \mathrm{MeV}$. For production of ${ }^{99} \mathrm{Tc}$ the optimum range is $8-35 \mathrm{MeV}$.

And it is also clear that the TALYS 1.6 nuclear reaction simulation code can be used safely if data is needed where there is no experimental data available.

\section{References}

[1] A. Kaplan, E. Tel, E.G. Aydın, A. Aydın, M. Yılmaz, Appl. Radiat. Isotopes 67, 570 (2009).

[2] S.M. Qaim, Radiochim. Acta 89297 (2001).

[3] B. Scholten, E. Hess, S. Takacs, Z. Kovacs, F. Tarkanyi, H.H. Coenen, S.M. Qaim, J. Nucl. Sci. Technol. 39, 1278 (2002).

[4] M.B. Chadwick, Radiochim. Acta 89, 325 (2001).

[5] A. Koning, S. Hilaire, S. Goriely, TALYS-1.6 A Nuclear Reaction Program, User Manual, NRG, The Netherlands 2013.

[6] EXFOR/CSISRS, Experimental Nuclear Reaction Data File, Brookhaven National Laboratory, National Nuclear Data Center, 2009. 